\title{
An X-ray View of Radio Millisecond Pulsars
}

\author{
Slavko Bogdanov and Jonathan E. Grindlay \\ Harvard-Smithsonian Center for Astrophysics, 60 Garden Street, Cambridge, MA 02138
}

\begin{abstract}
.
In recent years, X-ray observations with Chandra and XMM-Newton have significantly increased our understanding of rotation-powered (radio) millisecond pulsars (MSPs). Deep Chandra studies of several globular clusters have detected X-ray counterparts to a host of MSPs, including 19 in 47 Tuc alone. These surveys have revealed that most MSPs exhibit thermal emission from their heated magnetic polar caps. Realistic models of this thermal X-ray emission have provided important insight into the basic physics of pulsars and neutron stars. In addition, intrabinary shock X-ray radiation observed in "blackwidow" and peculiar globular cluster "exchanged" binary MSPs give interesting insight into MSP winds and relativistic shock. Thus, the X-ray band contains valuable information regarding the basic properties of MSPs that are not accesible by radio timing observations.
\end{abstract}

Keywords: Neutron stars, millisecond pulsars, X-rays PACS: $97.60 . \mathrm{Gb}, 97.60 . J \mathrm{~d}, 98.70 . \mathrm{Qy}$

\section{INTRODUCTION}

Rotation-powered ("recycled") millisecond pulsars (MSPs) were discovered at X-ray energies during the ROSAT all-sky survey [1]. In its lifetime, ROSAT detected X-ray emission from $10 \mathrm{MSPs}$ in the field of the Galaxy and one in the globular cluster M28 [2]. These observations established that MSPs are very faint $\mathrm{X}$-ray sources with typical $\mathrm{X}$-ray luminosities in the range $L_{X} \sim 10^{30-31} \mathrm{ergs} \mathrm{s}^{-1}$. In recent years, the superb sensitivity of the Chandrd and XMM-Newton X-ray observatories have permitted much more detailed studies of these objects. Chandra, in particular, with its unprecedented sub-arcsecond angular resolution, has proven to be ideally suited for studies of globular clusters, where MSPs are found in great abundance. In addition, both facilities have allowed detailed spectroscopic and timing studies of the nearby MSPs in the field of the Galaxy [3, 4]. Herein, we summarize the current state of affairs and examine future prospects in the X-ray study of MSPs.

\section{X-RAY STUDIES OF GLOBULAR CLUSTER MILLISECOND PULSARS}

Radio timing surveys have revealed that globular clusters are veritable treasure troves of MSPs [e.g., 5, 6]. As such, they allow a very efficient way to study MSPs at $\mathrm{X}$-ray energies due to their inherent faintness of these objects, which necessitates very long exposures. This is best examplified by the detection of X-ray counterparts to all 19 MSPs with known radio timing positions (Fig. 1) in a deep Chandra ACIS-S exposure of 47 Tuc. The large majority of the 47 Tuc MSPs (16 out of 19) appear to be soft, thermal X-ray sources with temperatures $T_{\text {eff }} \sim(1-3) \times 10^{6} \mathrm{~K}$, emission radii $R_{\text {eff }} \sim 0.1-3 \mathrm{~km}$, and luminosities $L_{X} \sim 10^{30-31} \operatorname{ergs~s}^{-1}$ [7]. The inferred effective emission areas imply that this radiation originates from the heated magnetic polar caps of the pulsar, as suggested by theoretical pulsar models [8, 9]. The thermal nature of the observed X-ray emission was subsequently confirmed with Chandra HRC-S timing observations, which revealed relatively broad pulsations with relatively low $(\leq 50 \%)$ pulsed fractions [10]. For several MSPs, there is indication of an additional thermal component (implying non-uniform heating of the polar cap), similar to what is seen in several nearby field MSPs [3]. These similarities in X-ray properties imply that, in general, no systematic differences exist between MSPs in globular clusters and those in the field of the Galaxy. It has been speculated that globular cluster MSPs should differ from those in the field of the Galaxy due to repeated dynamical binary exchanges and accretion episodes, which may alter the magnetic field topology of the pulsar from a dipolar to a multipolar one [11, 12]. However, the X-ray data indicates that even if most globular cluster MSPs do undergo "re-recycling", this does not significantly alter the observable properties of these objects.

1 http://cxc.harvard.edu/

2 http://xmm.vilspa.esa.es/ 


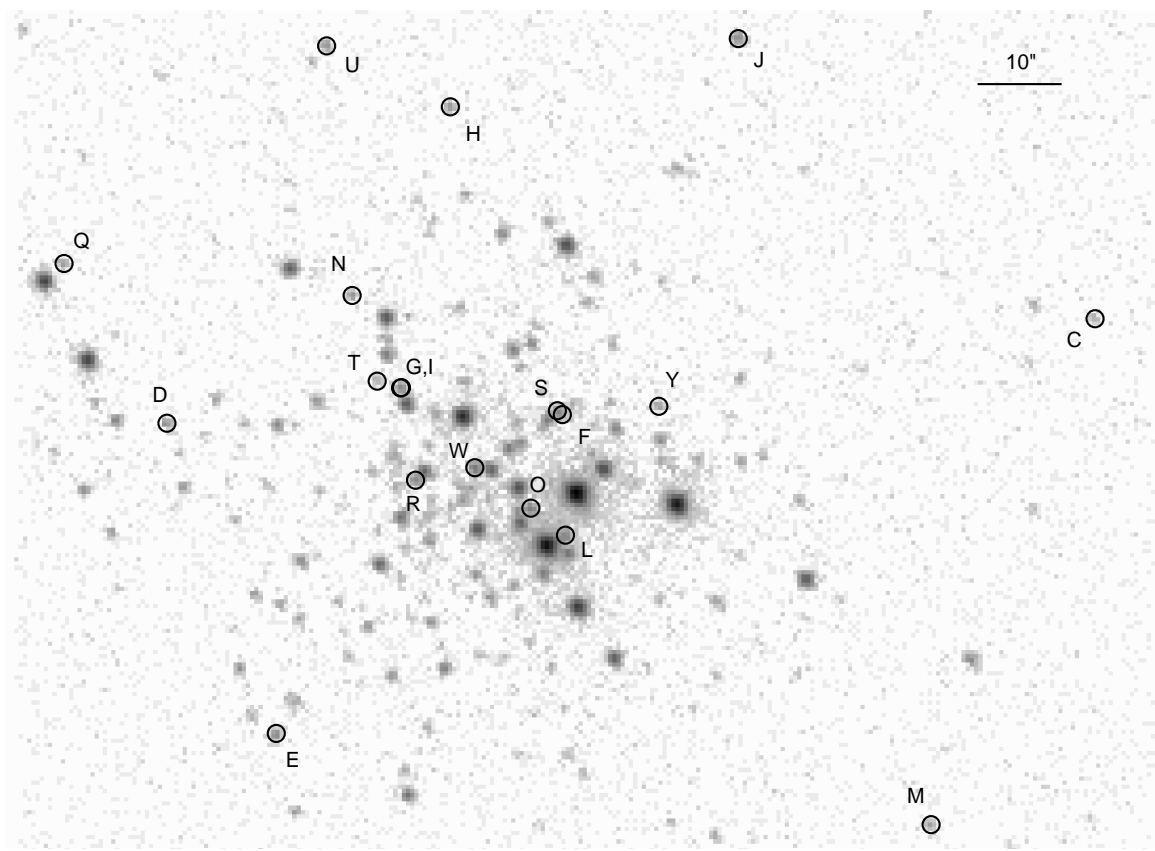

FIGURE 1. Chandra ACIS-S $0.3-6 \mathrm{keV}$ image of the core of the globular cluster 47 Tucanae. The 1" circles are centered on the radio timing positions of the 19 MSPs. An X-ray source is associated with the position of each MSP [see 7, for details].

\section{MODELING THERMAL X-RAY EMISSION FROM MILLISECOND PULSARS}

It has been shown that modeling X-ray spectroscopic and timing data of thermal MSPs can be used to extract important information regarding the properties of the neutron star [13, 14, 15]. Given that the vast majority of MSPs appear to be thermal X-ray sources, it is important to develop realistic theoretical models to ascertain whether this method can be employed to gain useful insight into the properties of MSPs. Such models of surface emission from MSPs need to incorporate general relativistic effects of gravitational redshift and bending of photon trajectories as well as special relativistic effects (Doppler boosting and aberration) due to the rapid motion of the stellar surface. It is also necessary to consider an optically-thick, weakly magnetized light-element atmosphere [see, e.g., 16]. Such an atmospheric layer is expected at the surface of a MSP given the standard formation scenaro of MSPs, involving accretion of a substantial amount of gas [17].

The synthetic pulse profiles of MSPs generated using these models are characterized by broad pulsations and moderate pulsed fractions, which are significantly larger than those of a blackbody emission spectrum for the same assumed parameters (see Fig. 2). Most notably, unlike the beamed radio emission, the duty cycle of the $\mathrm{X}$-ray radiation is always $100 \%$ and is observable for all combinations of $\alpha$ and $\zeta$. These characteristics have important implications for population studies of MSPs (see below).

A hydrogen atmosphere polar cap X-ray emission model has been found to be in fair agreement with the available X-ray data of the three nearest known MSPs, PSRs J0437-4715 [14, 18], J2124-3358, and J0030+0451 [19]. On the other hand, a blackbody emission model is inconsistent the observed pulse profiles. This provides compelling evidence that a gaseous atmosphere is indeed present at the surface of these neutron stars. For PSRs J0437-4715 and J2124-3358, the data indicates that the two polar caps are not diametrically opposite on the stellar surface, implying an off-center dipole magnetic axis. Perhaps more importantly, the thermal X-ray pulsations offers interesting constraints on the mass-to-radius ratio $(M / R)$ of the neutron star. In particular, for J0437-4715, J2124-3358, and J0030+0451 we find $R=6.9-10.6, R>7.2$, and $R>9.5 \mathrm{~km}$, respectively (68\% confidence), assuming $M=1.4 \mathrm{M}_{\odot}$.

Modeling of the thermal X-ray pulse profiles from MSPs also gives useful limits on the magnetic field geometry of the pulsar, which in turn provides stringest observational constraints on theoretical pulsar models. For example, Ruderman has postulated the presence of crustal "plates" on the NS surface formed by shear stresses on the crust caused by neutron superfluid vortex lines pinned to lattice nuclei. The motion of these plates would cause the magnetic fields of MSPs to mi- 


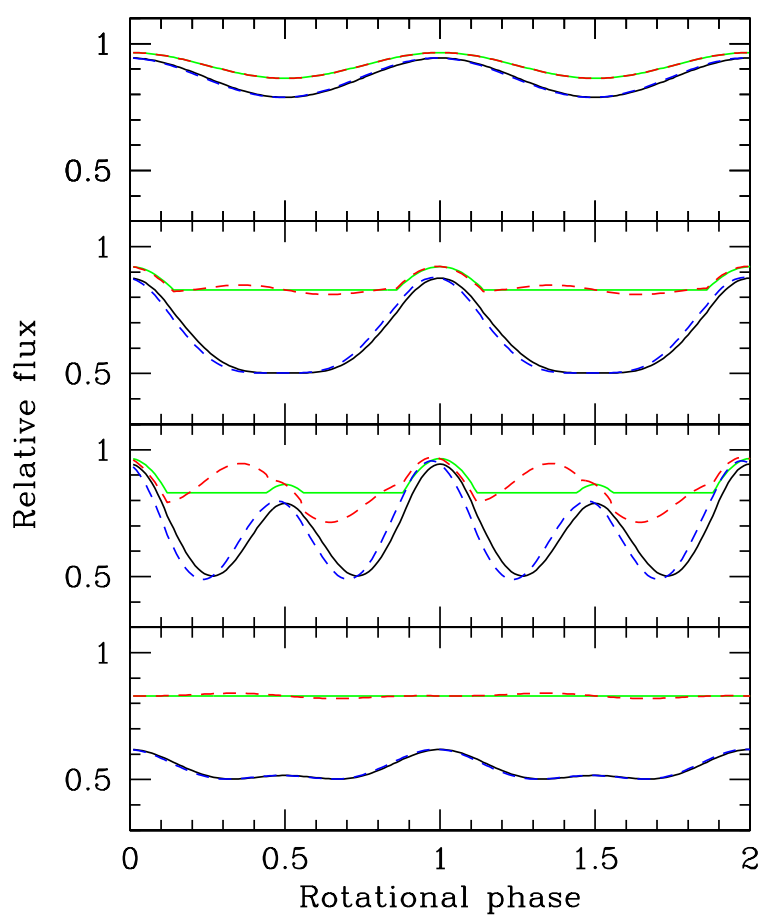

FIGURE 2. Representative hydrogen atmosphere model lightcurves for a rotating $M=1.4 \mathrm{M}_{\odot}, R=10 \mathrm{~km} \mathrm{NS}$ with two point-like antipodal hot spots [18]. The panels show pulse profiles for $\left(\alpha=10^{\circ}, \zeta=30^{\circ}\right),\left(\alpha=30^{\circ}, \zeta=60^{\circ}\right),\left(\alpha=60^{\circ}\right.$, $\left.\zeta=80^{\circ}\right)$, and $\left(\alpha=20^{\circ}, \zeta=80^{\circ}\right)$, from top to bottom, respectively. The solid lines in each plot correspond to a $\mathrm{H}$ atmosphere (black) and blackbody emission (green) with no Doppler effect included. The dashed lines show the effect of Doppler boosting and aberration for $P=4 \mathrm{~ms}$. All fluxes are normalized to the value for $\alpha=\zeta=0$.

grate across the stellar surface, resulting in either an aligned magnetic field or one pinched at one of the spin poles. However, the observed thermal pulse shapes and pulsed fractions from the nearest MSPs $(\sim 30-50 \%)$ suggest that the magnetic field resembles a (nearly) centered dipole and is moderately inclined with respect to the spin axis (see Fig. 3). This, in turn, implies that the dipole fields of these (and likely all) MSPs do not have a tendency to migrate towards the spin pole or align with the spin axis. Moreover, the infered X-ray-emitting areas are consistent with the classical polar cap area $\left[R_{p c}=(2 \pi R / c P)^{1 / 2} R \mathrm{~km}\right]$ and do not favor so-called "squeezed" polar caps [20].

\section{"BLACK WIDOW" AND "EXCHANGED” MSP BINARIES}

A number of radio eclipsing binary MSPs, such as the canonical "black widow" pulsar B1957+20 [21, 22], 47
Tuc J and $\mathrm{O}$ [7], and M71A [23], exhibit unresolved hard non-thermal X-ray emission. This is likely synchrotron radiation from a shock formed by the action of the pulsar wind on the companion star [24]. X-ray studies (complemented by optical observations) of these systems offer a useful diagnostic of the properties of MSP winds.

The eclipsing binary MSPs PSR J1740-5340 in the globular cluster NGC 6397 [25, 11] and PSR J00247204W in 47 Tuc [26, 27] exhibit very peculiar radio, optical, and X-ray properties that set them apart from other MSPs (see Fig. 4). The unusual behavior of these systems most probably stems from the fact that they are bound to unevolved (main-sequence-like) companions. This is at odds with the standard formation scenario of MSPs, which requires the companion to evolve off the main sequence in order for accretion and spin-up to commence [17]. It is likely that the current binary partners were not the stars that spun up the MSPs. As the dense environment of a globular cluster core is conducive to close dynamical encounters between stars, it is very likely that the present companion was acquired in a close binarybinary exchange encounter. These "exchanged" binaries offer a unique opportunity to study NSs transitioning from accretion to rotation power. In particular, the observed X-ray and optical properties of the radio MSP PSR J0024-7204W are remarkably similar to those of the low-mass X-ray binary and X-ray millisecond pulsar SAX J1808.4-3658 in quiescence. This supports the conjecture that the non-thermal X-ray emission and optical modulations seen in the SAX J1808.4-3658 system in a quiescent state are due to interaction between the wind from a reactivated rotation-powered pulsar and matter from the companion star. The striking similarities between the two systems provide further support for the long-sought connection between millisecond radio pulsars and accreting neutron star systems.

\section{NON-THERMAL X-RAY PULSATIONS}

A very small subset of MSPs show remarkably sharp Xray pulsations, indicative of non-thermal magnetospheric emission processes. These MSP include the exceptionally energetic PSRs B1937+21 [2], B1821-24 [29], and $\mathrm{J} 0218+4232\left(\dot{E}=2.4 \times 10^{35} \mathrm{ergs} \mathrm{s}^{-1}, B_{\text {surf }}=4.3 \times 10^{8}\right.$ $G)$ [28] as well as the mildly recycled relativistic binary MSP PSR J0737-3039A [30]. This striking difference in X-ray spectral properties compared to most other MSPs implies a substantially different physical regime in the pulsar magnetosphere. Models of pulsars suggest that the combination of spin properties of these four pulsars place them near the locus of theoretical curvature radiation (CR) pair-production death lines [31] on the $P-\dot{P}$ diagram. Therefore, in these of pulsars, pair-production in the magnetosphere probably occurs via curvature radia- 

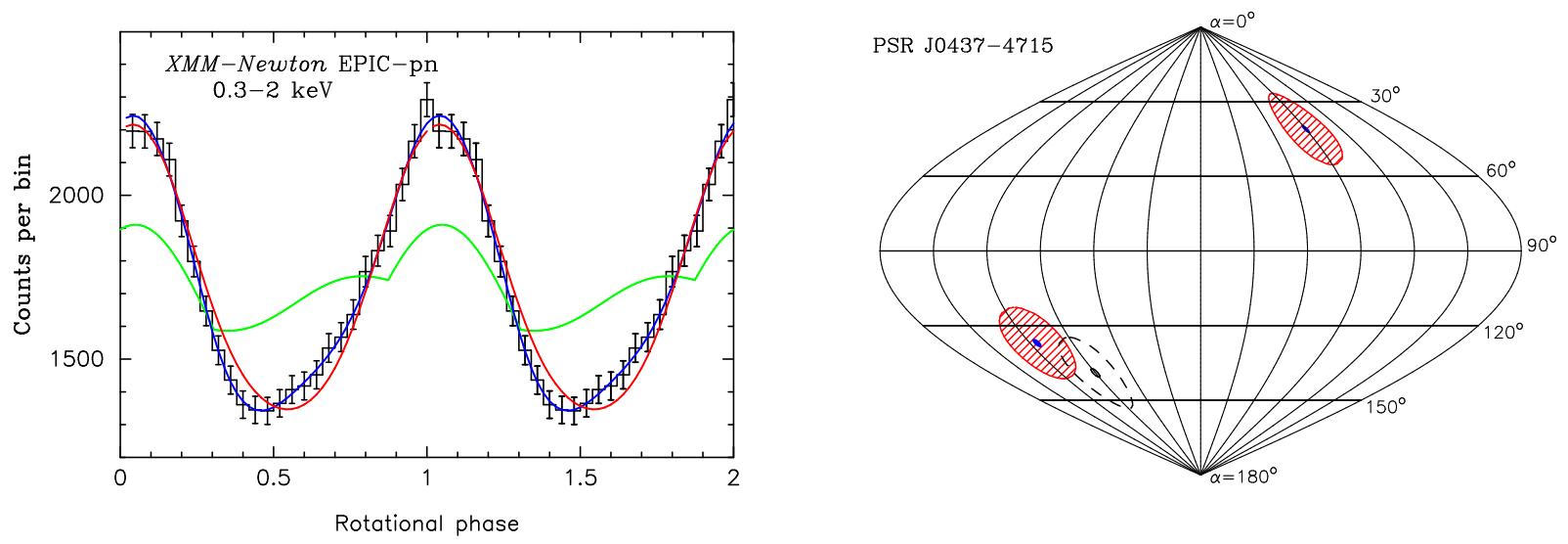

FIGURE 3. (Left) XMM-Newton EPIC-pn 0.3-2 keV pulse profile of PSR J0437-4715. The blue line shows the best fit curve for an off-center dipole field and a $\mathrm{H}$ atmosphere, while the green line is of a blackbody that best fits the spectrum of J0437-4715 for the same assumed compactness and geometry. The red line corresponds to the best fit $\mathrm{H}$ atmosphere model for a centered dipole (Right) Equal-area sinusoidal projection surface emission map of PSR J0437-4715 as inferred from the X-ray data for $R=10 \mathrm{~km}$ and $M=1.4 \mathrm{M}_{\odot}$. The dashed line shows the expected antipodal position of the secondary polar cap.

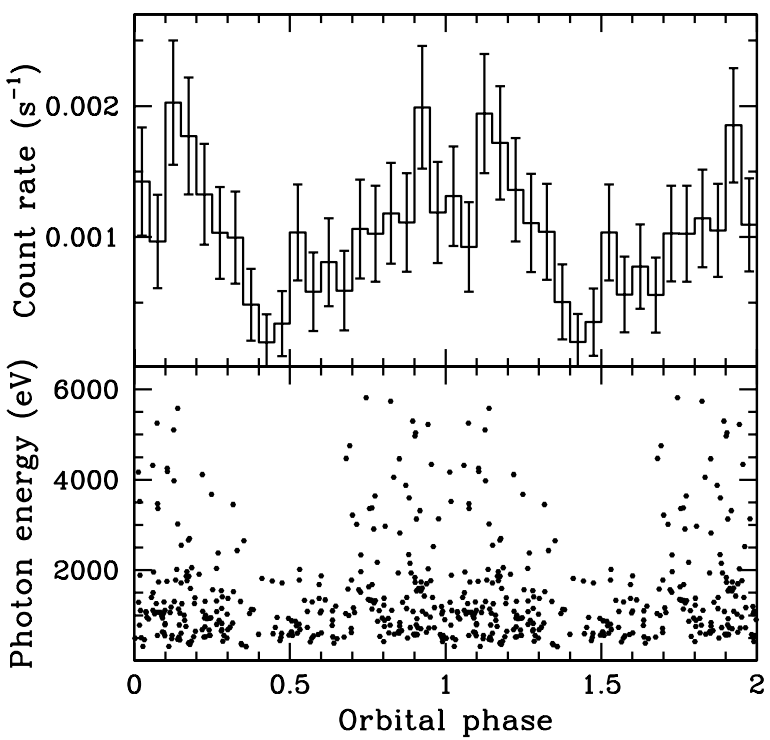

FIGURE 4. X-ray lightcurve of 47 Tuc $\mathrm{W}$ folded at the binary period $\left(P_{b}=3.2 \mathrm{~h}\right)$. Note the remarkable absence of hard X-ray photons between phases 0.4 and 0.7 , which can be explained by a geometric occultation of the intrabinary shock by the unusual main-sequence secondary star [see 27 , for details].

tion emitted by accelerating electrons. On the other hand, most MSPs (such as those in 47 Tuc), lie well below the theoretical CR pair production death lines. In these objects, inverse Compton scattering (ICS) is the primary $\mathrm{e}^{\mp}$ production mechanism and the conditions in the magne- tosphere favor substantial heating of the polar caps by a return flow of $\mathrm{e}^{+}[8]$.

\section{FUTURE PROSPECTS}

Due to the inherent faintness of MSPs, at present, only a handful of nearby objects can be studied in great detail [3]. The next generation of X-ray facilities, Constellation- $X$ and $X E U S$, are expected to have $\sim 100$ times greater sensitivity than which would enable studies of a much greater sample of MSPs. More importantly, these telescopes could be employed in blind timing searches for nearby $(<2 \mathrm{kpc})$ MSPs that are not observable at radio frequencies due to unfavorable viewing geometry. This is possible because the effect of light bending combined with the (nearly) antipodal configuration of the two MSP hot spots ensure that the thermal radiation is seen by all distant observers for any combination of $\alpha$ and $\zeta$. In contrast, at radio frequencies a pulsar is not observable if $|\alpha-\zeta|$ exceeds the opening half-angle $\rho$ of the radio emission cone.

Figure 5 shows the X-ray pulsed fraction of a $10 \mathrm{~km}$, $1.4 \mathrm{M}_{\odot} \mathrm{MSP}$ as a function of $\alpha$ and $\zeta$. Also shown are lines delineating the region of the $\alpha-\zeta$ plane for which a pulsar with a given radio emission cone width is observable at radio frequencies. If we assume a uniform distribution of pulsar obliquities $(\alpha)$ and viewing angles $(\zeta)$, for $\rho \simeq 30^{\circ}$ a substantial portion $(\sim 45 \%)$ of the MSP population is invisible to us in the radio. On the other hand, if we consider an X-ray timing survey with a limiting pulsed fraction sensitivity of $\sim 10 \%$, only $\sim 5-15 \%$ 


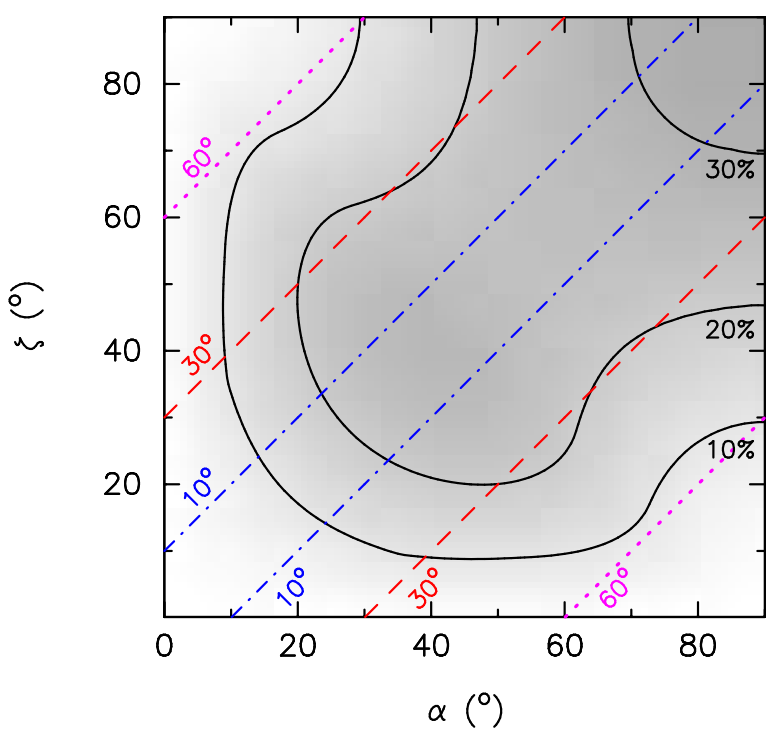

FIGURE 5. The $\alpha-\zeta$ plane for MSPs. The solid lines represent contours of constant thermal X-ray pulsed fraction for $R=10 \mathrm{~km}$ and $M=1.4 \mathrm{M}_{\odot}$. The dot-dashed, dashed, and dotted lines show $10^{\circ}, 30^{\circ}$, and $60^{\circ}$, respectively, opening halfangles of the radio emission cone.

(depending on the mass and radius of the star) of the MSPs will go undetected as pulsed sources though they will still be detected as X-ray sources [19]. The Galactic population of MSPs may in fact be preferentially clustered in a certain range of $\alpha$ due to the poorly understood effects of the accretion and magnetic field reduction processes during the LMXB phase on the NS. A deep X-ray timing survey of nearby $(<1-2 \mathrm{kpc})$ MSPs may, in principle, reveal whether this is indeed the case.

\section{CONCLUSIONS}

To date, nearly 40 MSPs have been detected at X-ray energies, 19 of which are found in the globular cluster 47 Tuc [7]. It has become apparent that X-ray spectroscopic and timing observations of MSPs represent a very promising approach towards elucidating important pulsar properties that are not measurable by other observational means such as radio timing observations. Further study of these sources will provide more important information regarding the X-ray properties of the Galactic population of MSPs, as well as valuable insight into the fundamental properties of NSs.

\section{ACKNOWLEDGMENTS}

We would like to thank G. Rybicki, C. Heinke, M. van den Berg, F. Camilo, P. Freire, A. Harding, and W.
Becker for their valuable contribution to the work described herein. We also extend our thanks to the conference organizers for their hospitality.

\section{REFERENCES}

1. W. Becker and J. Trümper, ApJ 365, 528-530 (1993).

2. W. Becker and J. Trümper, A\&A 341, 803-817 (1999).

3. V. E. Zavlin, ApJ 638, 951-962 (2006).

4. V. E. Zavlin, Ap\&SS 308, pp. 297-307 (2007).

5. F. Camilo, D. R. Lorimer, P. Freire, A. G. Lyne, and R. N. Manchester, ApJ 535, 975-990 (2000).

6. S. M. Ransom, J. W. T. Hessels, I. H. Stairs, P. C. C. Freire, F. Camilo, V. M. Kaspi, D. L. Kaplan, Science 307, 892-896 (2005).

7. S. Bogdanov, J. E. Grindlay, C. O. Heinke, F. Camilo, P. C. C. Freire, and W. Becker, ApJ 646, 1104-1115 (2006).

8. A. K. Harding and A. G. Muslimov, ApJ 568, 862-877 (2002).

9. L. Zhang and K. S. Chang, A\&A 398, 639-646 (2003).

10. P. B. Cameron, R. E. Rutledge, F. Camilo, L. Bildsten, S. M. Ransom, and S. R. Kulkarni, ApJ 660, 587-594 (2007).

11. J. E. Grindlay, F. Camilo, C. O. Heinke, P. D. Edmonds, H. Cohn, and P. Lugger, ApJ 581, 470-484 (2002).

12. K. S. Cheng and R. E. Taam, ApJ 598, 1207-1216 (2003).

13. G. G. Pavlov and V. E. Zavlin, ApJ 490, L91-L94 (1997).

14. V. E. Zavlin and G. G. Pavlov, A\&A 329, 583-598 (1998).

15. S. Bogdanov, J. E. Grindlay, and G. B. Rybicki, ApJ 648, L55-L58 (2006).

16. V. E. Zavlin, G. G. Pavlov, Yu. A. Shibanov, $A \& A$ 315, 141-152 (1996).

17. D. Bhattacharya and E. P. J. van den Heuvel, Phys. Reports 1, 1-124 (1991).

18. S. Bogdanov, G. B. Rybicki, and J. E. Grindlay ApJ, in press, astro-ph/0612791 (2007).

19. S. Bogdanov, J. E. Grindlay, A. K. Harding, G. B. Rybicki, ApJ, in press.

20. M. Ruderman, ApJ 379, 69-73 (1991).

21. B. Stappers, B. M. Gaensler, V. M. Kaspi, M. van der Klis, and W. H. G. Lewin Science 299, 1372-1374 (2003).

22. H. H. Huang and W. Becker, $A \& A$ 463, L5-L8 (2007).

23. R. F. Elsner, K. Ghosh, D. A. Swartz, et al., in preparation.

24. J. Arons and M. Tavani, ApJ 403, 69-73 (1993).

25. N. D'Amico, A. Possenti, R. N. Manchester, J. Sarkissian, A. G. Lyne, and F. Camilo, ApJ 561, L89-L92 (2001).

26. P. D. Edmonds, R. L. Gilliland, F. Camilo, C. O. Heinke, and J. E. Grindlay, ApJ 579 741-751 (2003).

27. S. Bogdanov, J. E. Grindlay, and M. van den Berg, ApJ 630, 1029-1036 (2005).

28. N. A. Webb, J.-F. Olive, and D. Barret, $A \& A$ 417, 181-188 (2004).

29. R. E. Rutledge, D. W. Fox, S. R. Kulkarni, B. A. Jacoby, I. Cognard, D. C. Backer, and S. S. Murray, ApJ 613, 522-531 (2004).

30. S. Chatterjee, B. M. Gaensler, A. Melatos, W. F. Brisken, and B. W. Stappers, ApJ, in press, astro-ph/0703181 (2007).

31. A. K. Harding, A. G. Muslimov, and B. Zhang, ApJ 576, 366-375 (2002).

32. M. Kramer, K. M. Xilouris, D. R. Lorimer, O. Doroshenko, A. Jessner, R. Wielebinski, et al., ApJ 501, 270-285 (1998). 